\title{
A!
}

This is an electronic reprint of the original article.

This reprint may differ from the original in pagination and typographic detail.

Pirkkalainen, Juha-Matti; Damskägg, E.; Brandt, M.; Massel, Francesco; Sillanpää, Mika

\section{Squeezing of Quantum Noise of Motion in a Micromechanical Resonator}

Published in:

Physical Review Letters

DOI:

10.1103/PhysRevLett.115.243601

Published: 11/12/2015

Document Version

Publisher's PDF, also known as Version of record

Please cite the original version:

Pirkkalainen, J-M., Damskägg, E., Brandt, M., Massel, F., \& Sillanpää, M. (2015). Squeezing of Quantum Noise of Motion in a Micromechanical Resonator. Physical Review Letters, 115(24), 1-15. [243601].

https://doi.org/10.1103/PhysRevLett.115.243601

This material is protected by copyright and other intellectual property rights, and duplication or sale of all or part of any of the repository collections is not permitted, except that material may be duplicated by you for your research use or educational purposes in electronic or print form. You must obtain permission for any other use. Electronic or print copies may not be offered, whether for sale or otherwise to anyone who is not an authorised user. 


\title{
乌ु
}

\section{Squeezing of Quantum Noise of Motion in a Micromechanical Resonator}

\author{
J.-M. Pirkkalainen, ${ }^{1}$ E. Damskägg, ${ }^{1}$ M. Brandt, ${ }^{1}$ F. Massel, ${ }^{2}$ and M. A. Sillanpää ${ }^{1, *}$ \\ ${ }^{1}$ Department of Applied Physics, Aalto University, P.O. Box 11100, FI-00076 Aalto, Finland \\ ${ }^{2}$ Department of Physics, Nanoscience Center, University of Jyväskylä, P.O. Box 35 (YFL), Jyväskylä FI-40014, Finland
}

(Received 15 July 2015; revised manuscript received 26 August 2015; published 7 December 2015)

\begin{abstract}
A pair of conjugate observables, such as the quadrature amplitudes of harmonic motion, have fundamental fluctuations that are bound by the Heisenberg uncertainty relation. However, in a squeezed quantum state, fluctuations of a quantity can be reduced below the standard quantum limit, at the cost of increased fluctuations of the conjugate variable. Here we prepare a nearly macroscopic moving body, realized as a micromechanical resonator, in a squeezed quantum state. We obtain squeezing of one quadrature amplitude $1.1 \pm 0.4 \mathrm{~dB}$ below the standard quantum limit, thus achieving a long-standing goal of obtaining motional squeezing in a macroscopic object.
\end{abstract}

The motion $x(t)=X_{1}(t) \cos \left(\omega_{m} t\right)+X_{2}(t) \sin \left(\omega_{m} t\right)$ of a harmonic oscillator having the natural oscillating frequency $\omega_{m}$ can be described by the quadrature amplitudes $X_{1}$ and $X_{2}$ which have slow fluctuations. The fluctuations, presented in units of the quantum zero-point fluctuation amplitude $x_{\mathrm{zp}}$, satisfy the Heisenberg uncertainty relation $\Delta X_{1} \Delta X_{2} \geq 1$. One of the two can be prepared (= squeezed) below the value 1 , at the expense of increased fluctuations in the other quadrature. In optics, squeezing of laser light was observed in early 1980s [1,2], not long after the possibility was realized.

It has been a formidable challenge to obtain squeezing in the motional state of a macroscopic object. The possibility of squeezing in the oscillations of massive gravitational antennae was hypothesized a long time ago [3,4], but technological limitations are too severe for experimental realization. Other motional quantum-mechanical phenomena, on the other hand, have recently been experimentally demonstrated $[5,6]$ in micromechanical resonators. The latter systems are nearly macroscopic in physical size, and therefore they provide an ideal test system for treating the borderline between quantum and classical. Of particular interest for these studies has been the cavity optomechanics setting coupling electromagnetic cavity mode and the oscillator motion [7]. Output of squeezed light [8-10] was recently observed, but this does not yet imply that the oscillator mode is squeezed.

Here we report the first realization of squeezing of the motional state of a nearly macroscopic body, realized as a micromechanical resonator measuring 15 microns in diameter. We utilize the novel idea of dissipative squeezing [11-13] [see Fig. 1(a)], where the system is allowed to cool towards a squeezed low-energy state. This method has the great advantage of being able to create unconditional squeezing in the steady state. This is in contrast with many other plausible methods of squeezing generation [14-20]. Our approach is closely related to the quantum nondemolition measurements [21-23] which, however, are not able to generate true squeezing without feedback. At this point we mention that classical squeezing of thermal noise is routinely observed in mechanical systems [24-26].

The mechanical element [Fig. 1(b)] is a drum resonator basically similar to Refs. [27,28]. The 120-nm-thick Al drum fabricated on a quartz substrate is connected through a narrow 70-nm vacuum gap to one end of the cavity. The latter consists [Fig. 1(c)] of a meandering superconducting $\mathrm{Al}$ strip. This kind of microwave optomechanical system is conveniently described using a model involving lumped electromagnetic elements. The interaction between the microwave cavity and the mechanical vibrations is given by the energy $g_{0} n_{P} x$, where $n_{P}$ is the number of photons externally applied by microwave pump(s), and $g_{0}$ is the radiation-pressure coupling energy. Because usually $g_{0}$ is much smaller than other energy scales, a large $n_{P} \gg 1$ is needed to effectively enhance the interaction up to a value $G=g_{0} \sqrt{n_{P}}$. If the pump is applied at the frequency $\omega_{-}=\omega_{c}-\omega_{m}$ (the red sideband), the physics leads to sideband cooling of the mechanical vibrations, possibly down to the quantum ground state $[28,29]$.

The scheme of Ref. [11] requires two pump microwaves, one applied at the red sideband and the other at the blue sideband frequency $\omega_{+}=\omega_{c}+\omega_{m}$ [Fig. 1(d)]. The two pertinent effective couplings are called $G_{-}$and $G_{+}$, respectively. This setup can be described as sideband cooling of a Bogoliubov (BG) mode [11,30], which in the laboratory frame corresponds to cooling the mechanical mode towards a squeezed vacuum state. The BG mode is defined by the annihilation operator $\beta \equiv b \cosh r+b^{\dagger} \sinh r$, obtained from the creation and annihilation operators $b^{\dagger}, b$ of the mechanical resonator. An arbitrary squeezing ratio $r$ can be selected by tuning the ratio of the two pumps: $\tanh r=G_{+} / G_{-}$. The BG mode-cavity system is described by the Hamiltonian $H=G_{\beta}\left(a^{\dagger} \beta+a \beta^{\dagger}\right)$ with the coupling energy $G_{\beta}=\sqrt{G_{-}^{2}-G_{+}^{2}}$. Here, $a^{\dagger}, a$ are the creation and 
(a)

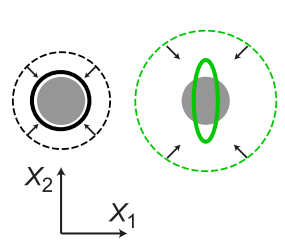

(b)
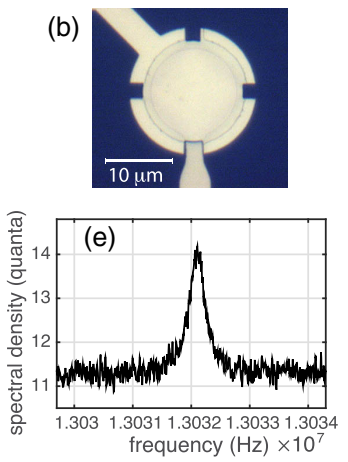

(c)

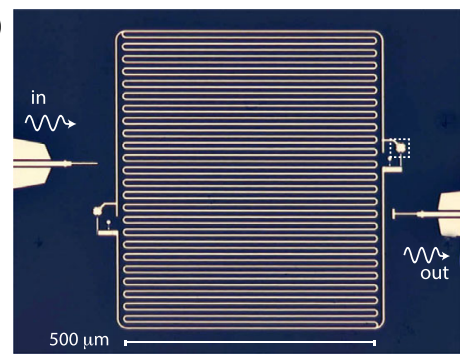

(d)

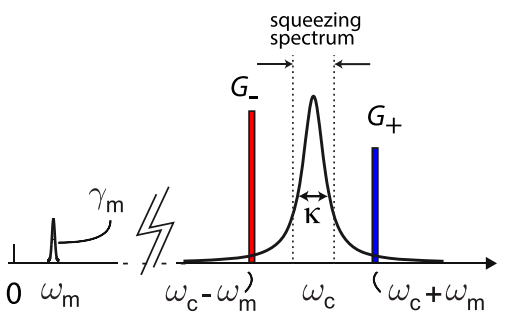

FIG. 1 (color online). Setup of the microwave optomechanical squeezing experiment. (a) Idea of dissipative squeezing. The initial fluctuation amplitudes of the quadratures are marked with dashed lines, and the final ones with solid lines. The gray circles denote the quantum ground state. In sideband cooling (left), the initial fluctuations uniformly cool towards the ground state. In a suitably engineered system (right), cooling can be quadrature dependent, hence leading to one quadrature becoming squeezed. (b, c) Optical micrographs of the micromechanical device and of the cavity. There are two drum resonators connected to the cavity; however, only one of them (within the dashed rectangle) operates. The cavity is asymmetrically coupled to the input port (left) and to the output port (right) for transmission measurements. (d) The frequencies involved in the scheme. The cavity is pumped by two nonequal-amplitude microwave tones at the sideband frequencies $\omega_{ \pm}=\omega_{c} \pm \omega_{m}$. (e) Example of the thermal motion signal measured at the refrigerator base temperature, with the coupling $G_{-} / 2 \pi \simeq 1.3 \mathrm{kHz}$.

annihilation operators of the cavity. Although a value $G_{-} / G_{+} \gtrsim 1$ would give rise to a high squeezing ratio, the effective coupling needed for cooling this mode would vanish. Hence, an optimum value is typically around $G_{-} / G_{+} \sim 1.5$.

The measurements are carried out inside a Bluefors dry dilution refrigerator in the temperature interval $7 \mathrm{mK} \ldots 200 \mathrm{mK}$. The cavity is first characterized having the frequency $\omega_{c} / 2 \pi \simeq 6.9004 \mathrm{GHz}$ and coupled to the transmission lines with the decay rates $\kappa_{E i} / 2 \pi \simeq$ $50 \pm 5 \mathrm{kHz}, \kappa_{E o} / 2 \pi \simeq 270 \pm 30 \mathrm{kHz}$ through the input and output ports, respectively. The internal losses are characterized by the rate $\kappa_{I} / 2 \pi \simeq 330 \pm 40 \mathrm{kHz}$, and the total cavity damping rate is $\kappa=\kappa_{E i}+\kappa_{E o}+\kappa_{I} \simeq$ $(2 \pi) \times 650 \pm 10 \mathrm{kHz}$. We operate in the good cavity limit $\omega_{m} / \kappa \simeq 20 \gg 1$, a prerequisite for efficient sideband cooling and squeezing generation.

The mechanical resonator is first characterized using a single pump tone at the red sideband. We choose very low pump powers such that the cavity backaction damping rate $\gamma_{-}=4 G_{-}^{2} / \kappa$ is much smaller than the intrinsic linewidth $\gamma_{m}$ of the mechanics. The emission at the cavity frequency then shows the usual thermal motion peak at the motional sideband at a frequency $\omega_{m} / 2 \pi \simeq 13.032 \mathrm{MHz}$ above the pump. We obtain $\gamma_{m} / 2 \pi \simeq 330 \mathrm{~Hz}$ corresponding to the $Q$ value $Q_{m} \simeq 3.9 \times 10^{4}$ from the data as in Fig. 1(e) $\left(\gamma_{-} \sim 12 \mathrm{~Hz}\right.$ was subtracted from the fit result).

An important benchmarking for cavity optomechanical experiments is how well the mechanical mode thermalizes to the temperature $T$ of the refrigerator. Here, we observe the linear temperature dependence expected [as seen in Fig. 2(a)] in equilibrium, $k_{B} T=n_{m}^{T} \hbar \omega_{m}$ down to $\simeq 25 \mathrm{mK}$. Here, $n_{m}^{T}$ is the equilibrium thermal phonon number defined accordingly. In what follows, we operate at the minimum $T$ where we know that the mechanical mode is at $25 \mathrm{mK}$ corresponding to $n_{m}^{T} \simeq 40$ at low pump powers.

We proceed with a series of further calibrations on the way towards demonstrating squeezing. Next, we perform a regular sideband cooling experiment $\left(G_{+}=0\right)$ (see, e.g., Refs. [31-35]). For calibrating $G_{-}$versus generator power, we study the peak width $\gamma_{m}+\gamma_{-}$as a function of power at modest values of $G_{-} \ll \kappa$. The most critical step, which also will account for most of the final imprecision, is to calibrate the system gain at the detection side. Each sideband cooling spectrum (about 20 curves at different power) are simultaneously fitted to theory with the same gain, using the $G_{-}$and $n_{m}^{T}$ just calibrated. For details, see Ref. [30]. We show examples of the sideband cooling spectra by the black symbols in Figs. 2(b)-2(e), overlaid with theoretical predictions from the standard formalism using input-output theory [30]. For the plot, we have subtracted a large background level due to the amplifier noise, hence displaying only the signal part due to the sample. We also find that the mechanical mode cools down to a thermal occupation $n_{m} \simeq 0.38\left(n_{m}=0\right.$ corresponds here to the ground state). The double peak seen in Fig. 2(e) signifies the onset of the strong-coupling regime when $G_{-} \sim \kappa$. The data are plotted in dimensionless units (quanta), which are the natural units from the theory point of view $\left(\mathrm{W} / \mathrm{Hz}\right.$ units are obtained by multiplying by $\left.\hbar \omega_{c}\right)$.

Given that we can cool the drum motion close to the ground state provides a promising starting point for creating squeezed motional states. We switch on the blue pump while keeping the red on, obeying $G_{-}>G_{+}$for stability. This creates a certain BG mode depending on the ratio $G_{-} / G_{+}$. In order to ascertain which BG mode we have, we calibrate the input line attenuations separately for both pump generators. We select $\gamma_{ \pm}=4 G_{ \pm}^{2} / \kappa \ll \gamma_{m}$ and 
(a)

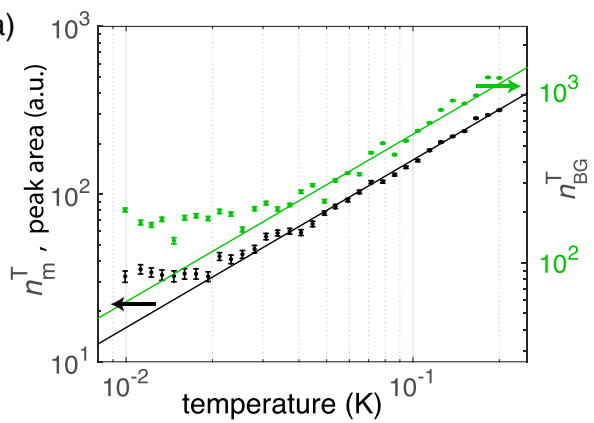

(b)

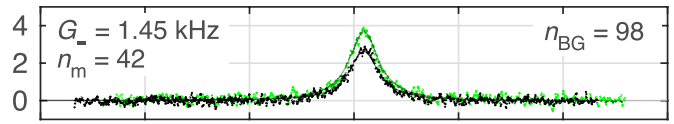

(c) $\begin{array}{lllllll}1.3029 & 1.303 & 1.3031 & 1.3032 & 1.3033 & 1.3034 & 1.3035\end{array}$

(c) 1.30

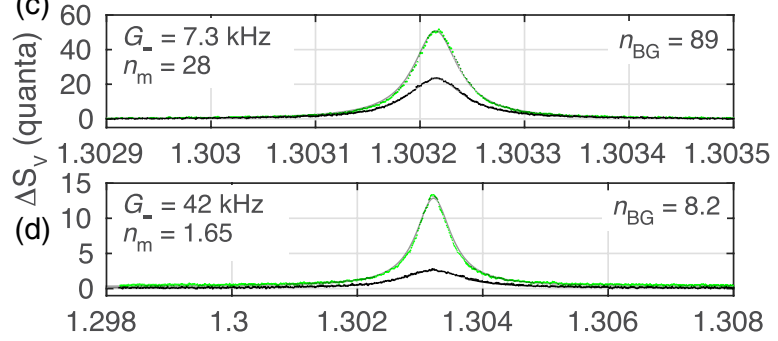

(e)

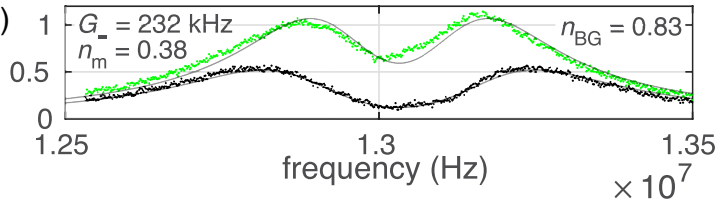

FIG. 2 (color online). Cooling the Bogoliubov mode. In all the panels, black refers to regular sideband cooling (i.e., $G_{+}=0$ ) used as calibration, whereas green refers to the $\mathrm{BG}$ mode experiment. (a) Thermalization in equilibrium. The left scale gives $n_{m}^{T}$, as well as the area of both the sideband cooling peaks, and of the BG mode peaks (both in a.u.), whereas the right-hand side scale is $n_{\mathrm{BG}}^{T}$. (b-e) Output spectrum during sideband cooling (black) or under BG mode conditions (green). The solid lines are theoretical curves. The $n_{m}$ values refer to the $G_{+}=0$ case.

adjust the powers such that we obtain equal response due to either pump. The imprecision is estimated to be $\pm 0.2 \mathrm{~dB}$ which is also the imprecision for constructing a given BG mode.

Next we discuss a specific BG mode obeying $G_{-} / G_{+} \simeq 1.52$, which is expected to represent a choice close to optimum. At the lowest pump powers, the backaction cooling is negligible, and we reveal the bare BG mode undressed from the cavity. Under this condition, the equilibrium BG mode occupancy $n_{\mathrm{BG}}^{T}$ is expected to follow a linear temperature dependence in the same way as the mechanical mode, but with an elevated temperature [30]. More relevant than $n_{\mathrm{BG}}^{T}$, however, is the agreement of the spectra with the theoretical prediction, which is connected here to the area under the Lorentzian BG mode peak. We test this in Fig. 2(a) and observe an excellent agreement with the theory. The green solid line is an expectation based on the calibrated $G_{-} / G_{+}$ratio and on the $G_{+}=0$ data.
Next, we increase the effective couplings. In order to mitigate possible gain drifts, we repeat a sequence of short measurements of the amplifier noise background, sideband cooling, and BG data. Plotted in conjunction with the sideband cooling data, in Figs. 2(b)-2(e) we display the BG mode spectra in green. The theoretical plots show a good agreement with the data. In Fig. 2(e), the BG mode curve is slightly shifted to the left, probably because of an energydependent shift in the cavity frequency. When using fixed pump frequencies as we do here, the cavity can become slightly detuned at certain pump powers. Here, we have used as adjustable parameters the bath temperatures of both the cavity and the mechanics. Both baths heat up with the pump powers, for the BG mode up to $n_{I} \simeq 0.74, n_{m}^{T} \simeq 86$ (instead of $n_{\mathrm{BG}}^{T}$, we prefer to quantify the bath with $n_{m}^{T}$ ) in Fig. 2(e), which is attributed to dielectric heating. These factors together set the limits for the cooling as well as squeezing. As one can anticipate, the baths heat up more if we apply both pumps instead of only one.

In Figs. 2(b)-2(e) we label the fitted values of the BG mode occupation $n_{\mathrm{BG}}$, which is a quantity analogous to $n_{m}$ in the case of regular sideband cooling. Although $n_{\mathrm{BG}}$ does not directly correspond to a physical temperature, a value $n_{\mathrm{BG}}<1$ as obtained in Fig. 2(e) is indicative of squeezing. We can more precisely infer the amount of squeezing by evaluating the quadrature variances [30]. This way, we obtain that the mechanical mode is squeezed below the vacuum level, i.e., in Fig. 2 (e) by $\simeq 1.1 \mathrm{~dB}$.

The best verification of squeezing comes from the quadrature spectra which amount to tomography of the state. We digitally mix down the signal using the center frequency $\omega_{c}$ of the pumps as a local oscillator (LO), hence making homodyne detection. The quadrature spectra show strong dependence on the LO phase. We identify the minimum emission as the $X_{1}$ quadrature and the maximum, offset by $90^{\circ}$, as $X_{2}$. In Fig. 3, we show the corresponding quadrature spectra $S_{1}$ and $S_{2}$ together with the total spectrum (the BG mode) and the cooling spectrum. We plot the quantities $2 S_{1}$ and $2 S_{2}$ for more conveniently presenting them together with the other two curves. The theoretical predictions overlaid on the data show an excellent agreement. In the best case of Fig. 3(d), the motion of the mechanical resonator has been squeezed about $1.1 \mathrm{~dB}$ below the Heisenberg limit. When varying the LO phase, we also observe an excellent agreement to theory (Fig. 4). The bath temperatures are found to be slightly enhanced over the previous data set in Fig. 2 [30]. We also find that these data agree with a slightly shifted BG ratio $G_{-} / G_{+} \simeq 1.43$ which is attributed to drift, not directly measured, in the tunable filters at room temperature over about one week after the calibration.

We now discuss an issue that is critical for understanding the quadrature spectra, namely, parametric effects beyond the ideal optomechanical model. One can satisfactorily model the total BG mode spectrum (and get equal squeezing) 

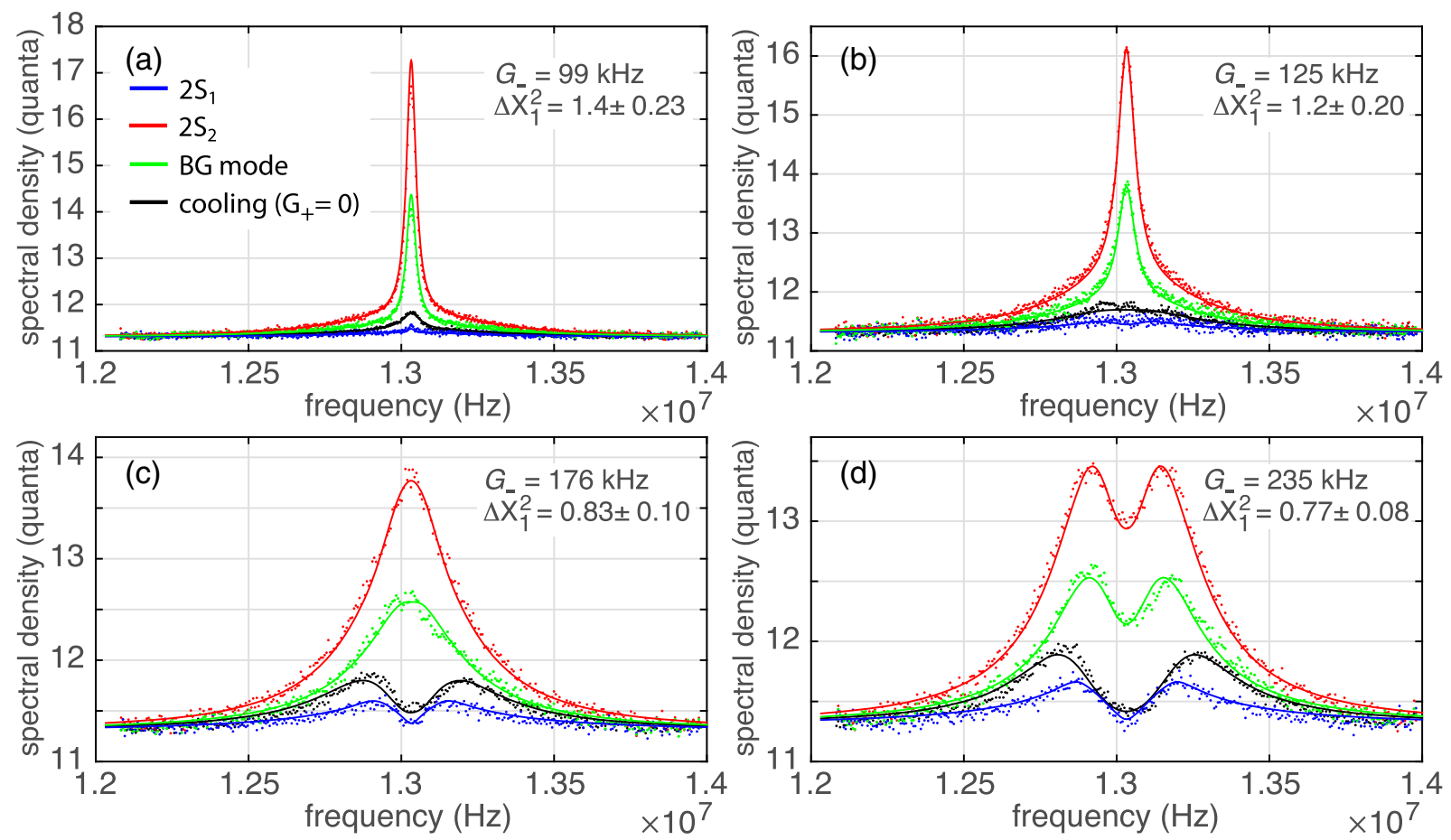

FIG. 3 (color online). Squeezing inferred from the quadrature spectra. In all panels, blue and red refer to the cold and hot quadratures $X_{1}$ and $X_{2}$, respectively. Black and green refer to the regular sideband cooling and the BG mode, respectively. The solid lines are theory curves. The pump powers are increased from (a) to (d) as marked in the panels, while the $G_{-} / G_{+} \simeq 1.43$ ratio is kept constant. The variances $\Delta X_{1}^{2}$ are marked, and a value less than 1 implies squeezing below vacuum. The amplitudes of parametric modulation to the cavity are $\epsilon_{c} / 2 \pi \simeq 35,48,49,56 \mathrm{kHz}$ from (a) to (d).

without imposing any parametric modulation [30]; however, the quadratures show much more phase dependence than predicted. In the scheme, mixing products of the pumps can appear both at $2 \omega_{m}$ and $2 \omega_{c}$, the frequencies most susceptible to causing parametric modulation via e.g. thermal effects or nonlinearities [22,30]. Modulation of $\omega_{c}$ out of phase with respect to the pumps gives an excellent match to the quadratures with the values quoted in Fig. 3. In order to verify the existence of this parametric effect, we carried out a measurement where we substantially detuned both pumps from the sideband resonance, by $\sim \pm 6 \kappa$, such that the center frequency stays at $\omega_{c}$ [30]. This high detuning essentially eliminates optomechanical phenomena, but a possible field at $2 \omega_{c}$ remains. Thus, we measure a spectrum consistent with a parametrically modulated oscillator with the correct phase. A possible explanation is nonlinear dissipation in the cavity [36] or a thermal effect. Although the parametric effects have a dramatic influence on the quadratures of the output spectrum, they only weakly affect the squeezing of the mechanics; in the present case, we find a reduction by about $10 \%$.

For the error analysis, we use a worst-case scenario of systematic errors from the calibrated parameters, and of direct statistical errors of the adjustable parameters. We find that the gain uncertainty is dominating. Because the shapes of the spectra are sensitive to most parameters, but squeezing is somewhat insensitive to any parameters in
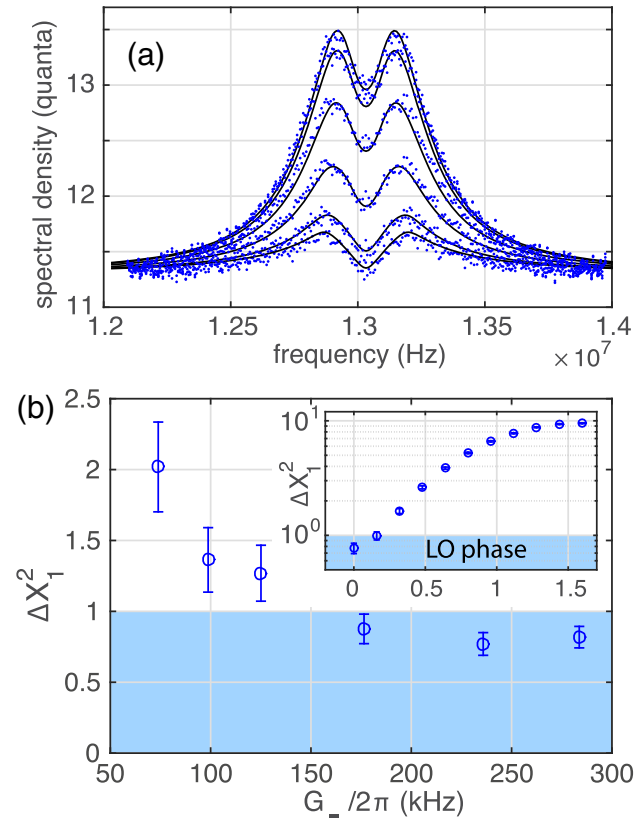

FIG. 4 (color online). Tomography and final results. (a) The quadrature data similar to Fig. 3(d), plotted at different LO phase values at $\pi / 10$ steps from 0 to $\pi / 2$, from bottom to top. (b) The $X_{1}$ quadrature variance as a function of pump power. The blue region signifies squeezing below the quantum limit. The inset shows the quadrature variance from the data in (a). 
the present parameter region, we obtain a relatively small imprecision of $\sim 10 \%$. The final numbers are quoted in Fig. 4(b).

In conclusion, we have achieved the reduction of the quantum fluctuations in a nearly macroscopic moving object below the "sound of silence" level by $1.1 \mathrm{~dB}$ in one quadrature. Following the discoveries of ground-state cooling [5,28,29], observation of zero-point fluctuations in mechanical systems [37], and entanglement [6], our work further establishes the reality of another fundamental quantum property in moving objects.

We would like to thank Pasi Lähteenmäki, Tero Heikkilä, Souvik Agasti, and André Xuereb for useful discussions. This work was supported by the Academy of Finland (Contracts No. 250280, No. CoE LTQ, No. 275245) and by the European Research Council (Grants No. 240387NEMSQED, No. 240362-Heattronics, and No. 615755CAVITYQPD). This work benefited from the facilities at the Micronova Nanofabrication Center and at the Low Temperature Laboratory infrastructure.

Note added.-Recently, we became aware of a similar experiment [38].

*Mika.Sillanpaa@aalto.fi

[1] D. F. Walls, Nature (London) 306, 141 (1983).

[2] R. E. Slusher, L. W. Hollberg, B. Yurke, J. C. Mertz, and J. F. Valley, Phys. Rev. Lett. 55, 2409 (1985).

[3] J. N. Hollenhorst, Phys. Rev. D 19, 1669 (1979).

[4] L. P. Grishchuk and M. V. Sazhin, Sov. Phys. JETP 57, 1128 (1983).

[5] A. D. O'Connell, M. Hofheinz, M. Ansmann, R. C. Bialczak, M. Lenander, E. Lucero, M. Neeley, D. Sank, H. Wang, M. Weides et al., Nature (London) 464, 697 (2010).

[6] T. A. Palomaki, J. D. Teufel, R. W. Simmonds, and K. W. Lehnert, Science 342, 710 (2013).

[7] M. Aspelmeyer, T. J. Kippenberg, and F. Marquardt, Rev. Mod. Phys. 86, 1391 (2014).

[8] D. W. C. Brooks, T. Botter, S. Schreppler, T. P. Purdy, N. Brahms, and D. M. Stamper-Kurn, Nature (London) 488, 476 (2012).

[9] A. H. Safavi-Naeini, S. Groblacher, J. T. Hill, J. Chan, M. Aspelmeyer, and O. Painter, Nature (London) 500, 185 (2013).

[10] T. P. Purdy, P.-L. Yu, R. W. Peterson, N. S. Kampel, and C. A. Regal, Phys. Rev. X 3, 031012 (2013).

[11] A. Kronwald, F. Marquardt, and A. A. Clerk, Phys. Rev. A 88, 063833 (2013).

[12] H. Tan, G. Li, and P. Meystre, Phys. Rev. A 87, 033829 (2013).

[13] D. Kienzler, H.-Y. Lo, B. Keitch, L. de Clercq, F. Leupold, F. Lindenfelser, M. Marinelli, V. Negnevitsky, and J. P. Home, Science 347, 53 (2015).

[14] M. Blencowe and M. Wybourne, Physica B (Amsterdam) 280, 555 (2000).
[15] M. J. Woolley, A. C. Doherty, G. J. Milburn, and K. C. Schwab, Phys. Rev. A 78, 062303 (2008).

[16] A. A. Clerk, F. Marquardt, and K. Jacobs, New J. Phys. 10, 095010 (2008).

[17] L. Tian, M. S. Allman, and R. W. Simmonds, New J. Phys. 10, 115001 (2008).

[18] K. Jähne, C. Genes, K. Hammerer, M. Wallquist, E. S. Polzik, and P. Zoller, Phys. Rev. A 79, 063819 (2009).

[19] A. Szorkovszky, A. C. Doherty, G. I. Harris, and W. P. Bowen, Phys. Rev. Lett. 107, 213603 (2011).

[20] G. Vasilakis, H. Shen, K. Jensen, M. Balabas, D. Salart, B. Chen, and E. S. Polzik, Nat. Phys. 11, 389 (2015).

[21] J. B. Hertzberg, T. Rocheleau, T. Ndukum, M. Savva, A. A. Clerk, and K. C. Schwab, Nat. Phys. 6, 213 (2010).

[22] J. Suh, M. D. Shaw, H. G. LeDuc, A. J. Weinstein, and K. C. Schwab, Nano Lett. 12, 6260 (2012).

[23] J. Suh, A. J. Weinstein, C. U. Lei, E. E. Wollman, S. K. Steinke, P. Meystre, A. A. Clerk, and K. C. Schwab, Science 344, 1262 (2014).

[24] D. Rugar and P. Grütter, Phys. Rev. Lett. 67, 699 (1991).

[25] R. Almog, S. Zaitsev, O. Shtempluck, and E. Buks, Phys. Rev. Lett. 98, 078103 (2007).

[26] I. Mahboob, H. Okamoto, K. Onomitsu, and H. Yamaguchi, Phys. Rev. Lett. 113, 167203 (2014).

[27] J. D. Teufel, D. Li, M. S. Allman, K. Cicak, A. J. Sirois, J. D. Whittaker, and R. W. Simmonds, Nature (London) 471, 204 (2011).

[28] J. D. Teufel, T. Donner, D. Li, J. W. Harlow, M. S. Allman, K. Cicak, A. J. Sirois, J. D. Whittaker, K. W. Lehnert, and R. W. Simmonds, Nature (London) 475, 359 (2011).

[29] J. Chan, T. P. M. Alegre, A. H. Safavi-Naeini, J. T. Hill, A. Krause, S. Gröblacher, M. Aspelmeyer, and O. Painter, Nature (London) 478, 89 (2011).

[30] See Supplemental Material at http://link.aps.org/ supplemental/10.1103/PhysRevLett.115.243601 for a detailed description of the experiment, theory, and data analysis.

[31] S. Gigan, H. R. Boehm, M. Paternostro, F. Blaser, G. Langer, J. B. Hertzberg, K. C. Schwab, D. Baeuerle, M. Aspelmeyer, and A. Zeilinger, Nature (London) 444, 67 (2006).

[32] A. Schliesser, P. Del'Haye, N. Nooshi, K. J. Vahala, and T. J. Kippenberg, Phys. Rev. Lett. 97, 243905 (2006).

[33] T. Rocheleau, T. Ndukum, C. Macklin, J. B. Hertzberg, A. A. Clerk, and K. C. Schwab, Nature (London) 463, 72 (2010).

[34] F. Massel, S. U. Cho, J.-M. Pirkkalainen, P. J. Hakonen, T. T. Heikkilä, and M. A. Sillanpää, Nat. Commun. 3, 987 (2012).

[35] X. Song, M. Oksanen, J. Li, P. J. Hakonen, and M. A. Sillanpää, Phys. Rev. Lett. 113, 027404 (2014).

[36] A. Eichler, J. Moser, J. Chaste, M. Zdrojek, I. Wilson-Rae, and A. Bachtold, Nat. Nanotechnol. 6, 339 (2011).

[37] A. H. Safavi-Naeini, J. Chan, J. T. Hill, T. P. M. Alegre, A. Krause, and O. Painter, Phys. Rev. Lett. 108, 033602 (2012).

[38] E. E. Wollman, C. U. Lei, A. J. Weinstein, J. Suh, A. Kronwald, F. Marquardt, A. A. Clerk, and K. C. Schwab, Science 349, 952 (2015). 